\section{A REVIEW ON SCHEDULING IN FLEXIBLE MANUFACTURIN SYSTEM USING HEURISTICS \\ APPROACH}

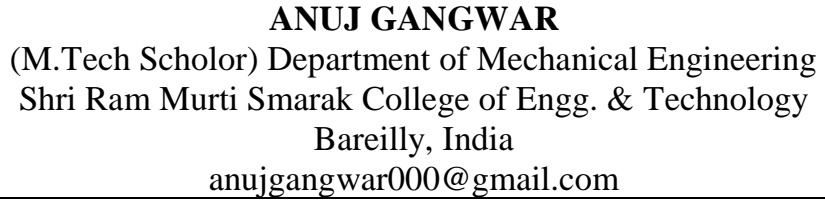

\author{
ASHWANI KUMAR \\ (Asst. Professor) Department of Mechanical Engineering \\ Shri Ram Murti Smarak College of Engg. \& Technology \\ Bareilly, India \\ ashipe90@gmail.com
}

\begin{abstract}
In recent years, Heuristics approaches involving natural computing techniques are recommended to resolve routing and sequencing combinatorial issues in versatile producing or FMS system. These techniques are applied one by one and in hybrid forms with relative success to planning issues. This work tries to review the achievement of heuristic approach applied in planning issues.
\end{abstract}

Keywords-Flexible Manufacturing System, Heuristic Approach, Sequencing, scheduling

\section{INTRODUCTION}

Over the last two decades, scheduling problems feature more prominently in flexible manufacturing system than in computing because manual or mathematically based solutions were sufficient. However, the growing complexity, flexibility, sequencing, line balancing of the scheduling problems and the advances in the development of high-speed computing devices fuelled the desire to push the boundaries of knowledge in this area. In the FMS scheduling different approaches used to schedule the system these are given below-
a. Simulation based approach
b. Artificial intelligence-based approach
c. Multi criteria decision making approach
d. Mathematical programming approach
e. Heuristic approach

In this paper we are using heuristic approach for schedule the FMS system. In this approach FMS programming drawback is solved in 2 stages: choosing the machine for every job operation

(routing) so determinative the process sequence for every machine. While the "routing than sequencing" strategy used in this approach.

The routing and sequencing downside involves the determination of the order of process job over machines to satisfy a desired objective whereas all jobs have a similar machine sequence. It's been one in every of the foremost researched topics within the programming literature. Among the required objectives, most of the eye has been dedicated to over machines make span minimization and to flow time minimization. Hence this introduction gives an overview of the scheduling problem in the FMS system. 


\section{Over view of Scheduling}

Scheduling is a very important concept for the FMS

system. Scheduling involves developing and managing a plan to achieve an objective in solving the general problem of satisfying time and resources constraints between a number of tasks. According to Sims,

"People face programming issues and opportunities everyday: at the aerodrome, somebody is to blame for distribution planes to gates; at a plumbing service, somebody is to blame of distribution jobs to machines, in a very centre, somebody is to blame of distribution bound calls to specifically competent personnel."

So the above lines gives a clear idea to achieve an objective or developing and managing a plan in FMS system, scheduling is more important.

\section{Heuristic Approach}

A heuristic may be a rule of thumb procedure that determines a "good-enough", satisfactory and possible answer at intervals bound constraints, however not essentially guarantees the most effective or best, answer to a haul. A decent heuristic is usually at interval of 100 percent of optimality, the number of errors is not known and degree of optimality is not known. Heuristic strategies supported priority rules for job shop programming drawback are not a convenience however a necessity for choosing that job is started $1^{\text {st }}$ and bound machine.

Some of principles used to scheduling issues are -

i. FIFO (First in First Out)

i. SPT (Shortest Process Time)

\section{ii. SLACK}

A. heuristic scheduling algorithm which makes a collection of dispatching rules. Dispatching rules are used to prioritise jobs on numerous resources (i.e. machines, material handling equipment, etc.). There is a good base of literature accessible on these rules, (1) dispatching rules may be used totally different in numerous ways that in varied scheduled environments and against different performance criteria. In FMS scheduling very often, heuristic approaches adopt "routing than sequencing" strategy. Dispatching rules may be used for sequencing operation on a machine when the task routes are fastened. The heuristic algorithm rules solve routing and sequencing sub issues of FMS at the same time by combining FOL and triton at operation level. During this algorithm rule 2 definitions area unit of importance:

1. Partial schedule creates Span (PSCS)

2. Eligible Operation Set (EOS)

1.1 The main components of a scheduling problem model

In their individual analysis Ali [2], Kim [3] and Framinan [4] identified a number of components that make up a typical problem model:

1. The properties of each job e.g. processing time, release time

2. The properties of the machines

3. The objective of the scheduling that the cost of the schedule is to be minimized 


\section{MEASURE OF PERFORMANCES}

A. Make span: Make span is the completion time of the last job to depart the system. The diminution of the make span time reduces total time of completion of a collection of jobs and therefore will increases the assembly rate.

B. Mean flow time: Flow time is that distinction between the completion time and the unharness time of job within the workplace that is the number of your time employment spends within the system. Total flow time is that the total of completion times of all jobs and mean flow time is that the average of total flow time. The diminution of flow time reduces the in-process inventory.

C. Machine idle time: Total idle time represents the in operative time of all the machines and mean idle time is that the average of total idle time. The diminution of idle time of machines will increases the effective utilization of machines.

D. Tardiness: Tardiness is that the timing of jobs that does not meet the maturity.

\section{REVIEW OF SELECTED TECHNIQUES}

Heuristic techniques are a collection of possibilities. The following section presents an overview of the most common heuristic techniques that are usually applied in solving routing and sequencing problem in scheduling.

- In paper [1] proposed two dispatching rules Flexible task last (FOL) and Earliest completing occasions (EFT) to solved partial overlapping machine problem in sequencing.
They used both approaches and combined together for solving this problem.

\section{A. Flexible task last (FTL)}

FTL is a composite of two rudimentary dispatching rules, longest preparing Time (LPT) and Least Flexible Job First (LFJ). LPT endeavors to put the shorter activity toward the finish of calendar and they utilized for adjusting the heap. LFJ chooses among the accessible activity the activity can be handled on the most modest number of machines. For the consolidate dispatching rules, FOL is displayed as a positioning articulation and that mix can be executed as the accompanying capacity:

$$
\mathrm{f}(\mathrm{j}, \mathrm{k})=\underline{\exp \{\mathrm{n}(\mathrm{j}, \mathrm{k}) / \mathrm{Q}\}}
$$

$1(\mathrm{j}, \mathrm{k})$

Where $f(j, k)$ is the ranking index of FOL, described the flexibility of operation $\mathrm{k}$ of job ; $\mathrm{n}(\mathrm{j}, \mathrm{k})$ is the quantity of machines in the device that can method the operation; $1(\mathrm{j}, \mathrm{k})$ is common processing time of the operation.

\section{B. Earliest Finishing Time}

At the point when the activity to be scheduled by FOL, EFT find a machine for the operation based on the finishing times of this operation on different machines. EFT selects the fast machines for an operation.

\section{The heuristic algorithm}

It solves routing and sequencing sub-problems by consolidating FTL and EFT at task level. In this calculation two standards are for the most part utilized: 


\section{ELK ASIA PACIFIC JOURNAL OF MECHANICAL ENGINEERING RESEARCH}

ISSN 2349-9368 (Online); EAPJMER/issn. 2349-9368/2016; Volume 4 Issue 2 (2018)

1. Partial timetable makes length (PTML)

2. Eligible activity set (EAS)

So, the general calculation comprises of two sections, activity determination (FTL principle) and portion (EFT rule) and they analyze the complexity of this algorithm using above both combinations and written as far as just number of jobs.

Hence viability of this calculation they contrasted proposed calculation and a variety of LPT dispatching rule and finished up their calculation enhances around $30 \%$ as far as the timetable make range

- In paper [2] they look at couple of late heuristic that are autonomously created and propose new heuristic. They begin with characterizing the match shrewd trade system. In the combine shrewd trade strategy, the situation of a couple of employments are traded and the adjustment in the destinations work processed. In the event that the trade decreased estimation of target work at that point occupations are kept in their new positions. In this they compared CDS, NEH, HO, WANG, RZ, and WY heuristics and combine to all then propose a new heuristic to minimize the total completion time.

\section{A. Heuristic evaluation}

For evaluate his heuristic they perform basically two basic steps:

\section{- Experimental Design}

In this they analyze the execution of heuristic calculation utilizing the FORTRAN dialect on a SUN SPARC Station 20. In this handling times on each machine were arbitrarily created from a discrete uniform conveyance and investigations are performed in two cases. In first case modest number of employments are considered and the heuristic arrangement contrasted and ideal arrangement. In second huge number of jobs considered and compared with best solution. In this comparison they measure average percentage error, standard deviation and number of times optimal solution is obtained.

\section{- Computational Result}

In this shown the result of experimental design evaluation the general rate blunder was somewhat influenced by the difference in information run for all heuristics. A similar perception holds for the most part for standard deviation and the level of the quantity of ideal arrangement.

At last they seen that the combine shrewd trade strategy enhances the mistake execution of heuristic with an irrelevant increment in CPU time.

Hence, they conclude the proposed heuristic gives better outcome analyzed than NEH heuristic and they apply in NEH, RZ, WY a couple shrewd trade strategy and discover five new heuristic (IH1, IH3, IH4, IH5, IH7). This system yields critical enhancement (about half for vast number of employments) with unimportant time.

Y. kim, D. lee, and C. yoon [3] proposed a twostage heuristic algorithm for part input sequencing in FMS. In this paper they divided this problem into two sub problem, the input part grouping problem and the sequencing problem. In this they propose an algorithm and solved by two-stage heuristic process. In two stage process is repeated 


\section{ELK ASIA PACIFIC JOURNAL OF MECHANICAL ENGINEERING RESEARCH}

ISSN 2349-9368 (Online); EAPJMER/issn. 2349-9368/2016; Volume 4 Issue 2 (2018)

until all parts are released into the FMS. They used a flowchart for the two-stage heuristic algorithm is given below:

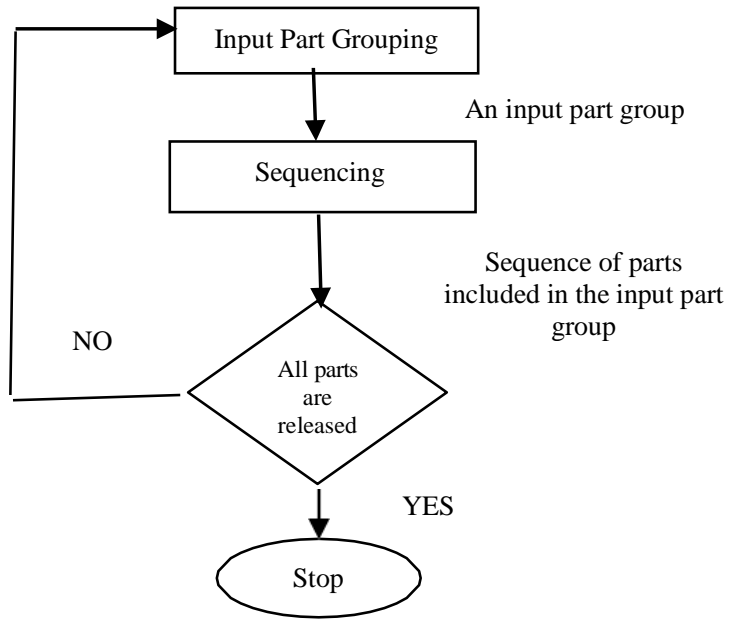

Fig. 1. An Overview of the two-stage heuristic algorithm

Therefore, in this paper researcher to solve input part grouping problem using two methods, static and dynamic ones. In static method identical input part groups are released into the Frame work over and over. So that is are keep up the discharged parts into the framework. In unique information part gathering strategy another info part bunch is resolved when all are parts assemble are discharged into the framework. So, the analyst to take care of this issue utilizing a few techniques that are:

\section{a. Minimal Part Set Method (MPS) \\ b. Expected remaining task at hand adjusting technique (EWB) \\ c. Expected aggregate remaining task at hand adjusting technique (ETWB \\ d. Expected residual outstanding task at hand adjusting technique (ERWB)}

To use over all techniques an info part amass is resolved, the information arrangement of the parts inside the info part gathering ought to be resolved. After illuminated this they take care of the sequencing issue with help of sixteen dispatching rules and got 64 input sequencing calculation.

\section{- Simulation experiment}

In this author compare the all algorithms and using direct release method in these sixteen dispatching rules without being partitioned into input part groups and direct release algorithm or also compared with two stage heuristic algorithms.

Hence, they conclude that the both problems are solved with the help of above methods and MPS given better performance of others.

J. Framinan and R. usano [4] proposed heuristic algorithm for flow shop sequencing. In this paper they investigate the execution of $\mathrm{NEH}$ heuristic concerning to minimization of make span time and then extend the heuristic algorithm for minimization the mean flow time.

They used NEH heuristic in two phases: first jobs are arranged in descending order with their processing time and in second phase, jobs sequence is arranged by evaluating the partial schedules originating from the initial order of the first phase. In this they after the NEH heuristic also propose two procedure for minimize the flow time that is-

1. Priori approach

2. Posteriori approach

In this they find out the posteriori approach are better than for the priori approach for minimization of mean flow and CPU time.

- In paper [5], H. Abedinnia et. al proposed new simple constructive heuristic algorithm in the 


\section{ELK ASIA PACIFIC JOURNAL OF MECHANICAL ENGINEERING RESEARCH}

ISSN 2349-9368 (Online); EAPJMER/issn. 2349-9368/2016; Volume 4 Issue 2 (2018)

permutation flow shop scheduling. In this they optimize the partial sequence at end of each iteration of NEH insertion phase with the help of both FL and LS heuristics and they improving the LS heuristics, which outperforms all other existing heuristics for finding total flow time in manufacturing system.

In this researcher to improve the Laha and Sarin heuristics and proposed a new heuristic that is AGB heuristics. In the AGB and LS heuristic the main important difference is given: remove job from the current partial sequence and insert it into the position of the remaining partial sequence and find out the total flow time for all new combinations.

In this paper to improve his heuristic researcher do again and again modification then they find out the proposed heuristic algorithm. For investigates the performance of new simple constructive heuristic they also perform the computational experiment.

\section{- Computational experiment}

In this they compare his proposed heuristic to the modified version of NEH and also compare the performance with the LS heuristic. Therefore, they conclude that the performance of any heuristic algorithm should be evaluated in two aspects that is effectiveness and the efficiency.

Hence, the author conclude that the modified heuristic algorithm is $24 \%$ improvement as compared to LS heuristic.

\section{CONCLUSION}

Hence, we conclude that the heuristic approach gives high quality and optimal solution in the scheduling problem. In this paper we observed, in the scheduling problem dispatching rules are more important and useful for minimize the make span and mean flow time as compared to others and also investigate the effectiveness and correctness of the heuristic algorithm. In this Sequencing and routing is the best option to reduce the make span and flow time or find the feasible and optimum solution.

\section{REFERENCES}

[1] Hamada Ghenniwa and Chun Wang, "Heuristic Scheduling Algorithm for Flexible Manufacturing System with Partially Overlapping Machine Capabilities," in proceeding of the IEEE, International Conference on Mechatronics \& Automation, July 2005.

[2] Ali Allahverdi and Tariq Aldowaisan, "New heuristics to minimize total completion time in $\mathrm{m}$ machine flowshop," International journal of Production Economics, vol. 77, pp. 71-83, 2002.

[3] Yeong-Dae Kim, Dong-Ho lee \& Chi-moon Yoon, "Two-stage heuristic algorithm for part input sequencing in flexible manufacturing systems," European Journal of Operation Research, vol. 133, pp. 624-634, 2001.

[4] Jose M. Framinan, Rainer Leisten \& Rafael Ruiz Usano, "Efficient heuristics for flowshop sequencing with the objectives of makespan and flowtime minimisation," European Journal of Operation Research, vol. 141, pp. 559-569, 2002.

[5] Hamid Abedinnia, Christoph H. Glock \& Andreas Brill, "New simple constructive heuristic algorithm for minimizing total flow time in the permutation flowshop scheduling 